\title{
Antioxidant Properties of Tonsil-Derived Mesenchymal Stem Cells on Human Vocal Fold Fibroblast Exposed to Oxidative Stress
}

\author{
Sung-Chan Shin $\mathbb{D}^{1},{ }^{1}$ Hyung-Sik Kim $\mathbb{D}^{2,3}$ Yoojin Seo ${ }^{(D)}{ }^{3}$ Cho Hee Kim, ${ }^{1}$ Ji Min Kim, ${ }^{1}$ \\ Hyun-Keun Kwon, ${ }^{3}$ Jin-Choon Lee, ${ }^{4}$ Eui-Suk Sung, ${ }^{4}$ and Byung-Joo Lee ${ }^{1}{ }^{1}$ \\ ${ }^{1}$ Department of Otorhinolaryngology, Head and Neck Surgery, Pusan National University School of Medicine, Pusan National \\ University and Biomedical Research Institute, Pusan National University Hospital, Busan 49241, Republic of Korea \\ ${ }^{2}$ Department of Life Science in Dentistry, School of Dentistry, Pusan National University, Yangsan, \\ Gyeongsangnam-do 50612, Republic of Korea \\ ${ }^{3}$ Dental and Life Science Institute, Pusan National University, Yangsan, Gyeongsangnam-do 50612, Republic of Korea \\ ${ }^{4}$ Department of Otorhinolaryngology, Head and Neck Surgery, Pusan National University School of Medicine, \\ Biomedical Research Institute, Pusan National University Yangsan Hospital, Yangsan, Gyeongsangnam-do 50612, Republic of Korea
}

Correspondence should be addressed to Byung-Joo Lee; voiceleebj@gmail.com

Received 26 July 2019; Revised 22 November 2019; Accepted 21 December 2019; Published 3 February 2020

Academic Editor: Hugo Guerrero-Cazares

Copyright (c) 2020 Sung-Chan Shin et al. This is an open access article distributed under the Creative Commons Attribution License, which permits unrestricted use, distribution, and reproduction in any medium, provided the original work is properly cited.

\begin{abstract}
The therapeutic potential of tonsil-derived mesenchymal stem cells (TMSCs) has been proved in several in vitro and in vivo models based on their antioxidative capacity. Oxidative stress is involved in the formation of vocal fold scars and the aging of vocal folds. However, few studies have examined the direct correlation between oxidative damage and reconstitution of extracellular matrix (ECM) in the vocal fold fibrosis. We, therefore, sought to investigate the impact of oxidative stress on cell survival and ECM production of human vocal fibroblasts (hVFFs) and the protective effects elicited by TMSCs against oxidative damages in hVFFs. hVFFs were exposed to different concentrations of tert-butyl hydroperoxide in the presence or absence of TMSCs. Cell viability and reactive oxygen species (ROS) production were assessed to examine the progression of oxidative stress in vitro. In addition, expression patterns of ECM-associated factors including various collagens were examined by real-time PCR and immunocytochemical analysis. We found that both cell viability and proliferation capacity of hVFFs were decreased following the exposure to $\mathrm{tBHP}$ in a dose-dependent manner. Furthermore, tBHP treatment induced the generation of ROS and reactive aldehydes, while it decreased endogenous activity of antioxidant enzymes in hVFF. Importantly, TMSCs could rescue these oxidative stress-associated damages of hVFFs. TMSCs also downregulated tBHP-mediated production of proinflammatory cytokines in hVFFs. In addition, coculture with TMSC could restore the endogenous matrix metalloproteinase (MMP) activity of hVFFs upon tBHP treatment and, in turn, reduce the oxidative stress-induced ECM accumulation in hVFFs. We have, therefore, shown that the changes in hVFF proliferative capacity and ECM gene expression induced by oxidative stress are consistent with in vivo phenotypes observed in aging vocal folds and vocal fold scarring and that TMSCs may function to reduce oxidative stress in aging vocal folds.
\end{abstract}

\section{Introduction}

Reactive oxygen species (ROS) are constantly produced in cells and are regulated by various intracellular antioxidant mechanisms under normal conditions. However, excessive production of ROS impairs cellular defense mechanisms, leading to the development of various diseases including diabetes, inflammatory conditions, cancer, and neurodegenerative diseases $[1,2]$. ROS are also involved in wound healing and aging of tissues. Although ROS are involved in defense against pathogens and have essential roles as mediators of cellular signaling, excessive amounts of ROS can hinder normal wound healing [3-5]. ROS are produced, predominantly by inflammatory cells, during the early stage of 
vocal fold wound healing [6]. Furthermore, oxidative stress markers have been found to be upregulated, and the composition of the extracellular matrix (ECM) was altered, in aging vocal folds [7]. However, the oxidative stress-mediated mechanisms responsible for these ECM changes in vocal fold lamina propria remain unclear.

Scarring of the vocal fold is an important clinical challenge that is not fully understood within the field of laryngology. Vocal cord scarring acts to significantly reduce the voice quality and eventually reduces the quality of life for patients. Several growth factors, such as hepatocyte growth factors (HGF), transforming growth factor, beta 3 (TGF $\beta-3$ ), and basic fibroblast growth factors (bFGF), have been used as preventative and therapeutic agents for vocal fold scars [8-10]. Moreover, several studies have investigated the therapeutic potential of mesenchymal and embryonic stem cells for the prevention and treatment of vocal fold scarring $[11,12]$. Injection of stem cells into the vocal fold wound may serve to improve healing and decrease the accumulation of collagen, thereby preventing scar formation in vocal folds. However, the precise mechanism responsible for the observed alterations in vocal fold ECM following injection of stem cells is not yet clear.

MSCs from various sources are also ideal candidates for cell-based and regenerative therapies in several fields due to their self-renewal capacity and multipotent properties [12-14]. In addition to direct regenerative properties, MSCs have been reported to exert various supportive functions including enhanced proliferation and differentiation during the process of tissue healing [15]. Through the paracrine production of various growth factors and cytokines, MSCs are also involved in a myriad of additional functions including angiogenesis, immunomodulation, and antiapoptotic regulation [16]. Recently, it has been reported that MSCs also elicit antioxidant properties against oxidative stress [16, 17].

However, MSCs derived from only certain tissues are commonly employed in in vitro tissue culture studies. Specifically, tonsil-derived mesenchymal stem cells (TMSCs) are readily obtained from tonsillectomy tissue, which is a common operation in otolaryngology. TMSCs have the ability to differentiate into various mesodermal cells including osteoblasts, chondrocytes, and adipocytes [18]. TMSCs also have the advantage of being able to rapidly proliferate and sustain up to 20 passages of cell expansion compared to MSCs obtained from other sources $[19,20]$.

To date, no study has examined the changes that occur within vocal fold fibroblasts following exposure to oxidative stress or the protective effect elicited by MSCs. Therefore, this study sought to investigate the changes that occur in the proliferative capacity of human vocal fold fibroblasts (hVFFs) as well as the expression patterns of ECM-related genes within these cells following exposure to oxidative stress. We also evaluated the antioxidant properties of TMSCs in hVFFs.

\section{Materials and Methods}

2.1. Human Vocal Fold Fibroblasts (hVFFs) and Human Tonsil-Derived Mesenchymal Stem Cells (TMSCs). The hVFFs from one donor were kindly provided by Professor Susan
Thibeault at the University of Wisconsin, USA. The cells were grown in Dulbecco's modified Eagle medium (DMEM, Invitrogen, Carlsbad, CA, USA) supplemented with $2 \mathrm{mM}$ L-glutamine, $100 \mathrm{mg} / \mathrm{mL}$ penicillin-streptomycin, $2.5 \mathrm{mg} / \mathrm{L}$ amphotericin B, and 10\% heat-inactivated fetal bovine serum (FBS, Gibco, Grand Island, NY, USA). Cells were maintained at $37^{\circ} \mathrm{C}$ in a humidified atmosphere containing $5 \% \mathrm{CO}_{2}$. TMSCs were isolated and characterized as described previously [1]. We used the cells that we validated the characteristics of mesenchymal stem cells by determining the differentiation potential and immunological phenotypes as we previously reported (Supplementary Figure 1) [18-20]. Briefly, TMSCs were isolated from excised palatine tonsil tissue obtained after tonsillectomy. Isolated tissues were washed and digested using $0.075 \%$ collagenase type I (Sigma, St. Louis, MO) for 30 minutes at $37^{\circ} \mathrm{C}$. The pellet was harvested and filtered through a $100 \mu \mathrm{m}$ nylon mesh. Cells were incubated overnight in $\alpha$-MEM containing $10 \%$ fetal bovine serum (FBS) at $37^{\circ} \mathrm{C}$ with $5 \% \quad \mathrm{CO}_{2}$, and adherent cells were further maintained. All procedures using human tonsil tissue-derived cells were conducted in accordance with guidelines approved by the Pusan National University Hospital Institutional Review Board. For this study, MSCs derived from tonsil tissues of two different patients were used.

2.2. Cell Proliferation and Viability Assays. Cell viability was determined using the CCK-8 assay. Briefly, hVFFs $\left(5 \times 10^{4}\right.$ cells/well $)$ in a logarithmic growth phase were seeded in 96-well plates and treated different concentrations of exogenous tBHP $(0,300,500,1000$, or $2000 \mu \mathrm{M})$ for $3 \mathrm{~h}$; $10 \mu \mathrm{L}$ of CCK- 8 dye (Abcam, Cambridge, MA USA) was then added into each well. The plates were incubated at $37^{\circ} \mathrm{C}$ for $2 \mathrm{~h}$. The optical density (OD) was read at $450 \mathrm{~nm}\left(\mathrm{Gen} 5^{\mathrm{TM}}\right.$ Microplate Reader, Winooski, VT, USA). Positively stained cells were considered viable and were presented as percentages of the control cells. The goal of this assay was to determine which conditions permitted up to $80 \%$ survival to establish an effective tBHP damage model. To evaluate the cell proliferation rate, the control and TMSC-cocultured hVFFs (as described below) were treated with $100 \mu \mathrm{M}$ of EdU for 3 hours with or without tBHP; then, the EdU uptake level of each group was determined using a Click-iT EdU flow cytometry assay kit (Invitrogen, Carlsbad, CA, USA) as per the manufacturer's instruction.

2.3. Coculture System. The hVFFs were seeded at an initial density of $4 \times 10^{5}$ cells $/ \mathrm{cm}^{2}$ in 6 -well culture plates. Transwells (upper chamber) were placed in other 6-well culture plates and seeded with different hVFF:TMSC ratios $\left(4 \times 10^{5}\right.$ hVFFs in lower chamber, $2 \times 10^{5}$ or $8 \times 10^{4}$ TMSCs in upper chamber). After cell attachment and stabilization, transwells containing TMSCs (for coculture) were transferred into the corresponding wells of the 6-well culture plate containing hVFFs to create the hVFF/TMSC coculture system without direct cell-to-cell contact. After 24 hours, transwell containing TMSCs was removed and hVFFs were used for further analysis. 
2.4. Assessment of ROS Production. The level of ROS induced by tBHP in hVFFs was measured using $2^{\prime}, 7^{\prime}$-dichlorofluorescein diacetate (DCFDA) as a fluorescent probe (Invitrogen). DCFDA is a fluorogenic dye that measures hydroxyl, peroxyl, and other ROS activities within the cell. After coculturing of hVFFs with TMSCs for $24 \mathrm{~h}$, transwells (upper chamber) containing TMSCs were removed and hVFFs were treated with tBHP, followed by the washing and incubation with DCFDA $(10 \mathrm{mM})$ for $45 \mathrm{~min}$ at $37^{\circ} \mathrm{C}$ in the dark. DCFDA solution was then removed, and the cells were washed twice with PBS. Absorbance was then measured using a fluorescence plate reader at $\mathrm{Ex} / \mathrm{Em}=485 / 535 \mathrm{~nm}$ in the endpoint mode (Gen5 ${ }^{\mathrm{TM}}$ Microplate Reader, Winooski, VT, USA).

\subsection{Quantification of Intracellular MDA and Measurement of} the Level of Antioxidant Activity. hVFFs and cocultured cellular malondialdehyde (MDA) were measured according to the manufacturer's instructions using a Lipid Peroxidation (MDA) Assay Kit (Abcam, Cambridge, MA USA). Briefly, under acidic conditions, MDA reacted with thiobarbituric acid (TBA) at $95^{\circ} \mathrm{C}$. The MDA-TBA conjugate was measured at $532 \mathrm{~nm}$ using a multimode microplate reader (Synergy H1 Hybrid reader). Intracellular MDA was expressed as $\mathrm{nmol} / \mathrm{mg}$ of cellular protein. Intracellular enzyme extracts were obtained by removing the supernatants and trypsinizing the hVFFs to collect them. The cells were then homogenized in a solution containing $10 \mathrm{mM}$ Tris- $\mathrm{HCl}$ buffer. The homogenate was centrifuged at $4^{\circ} \mathrm{C}, 3000 \mathrm{rpm}$ for $10 \mathrm{~min}$, and the resulting supernatant was collected for detection of superoxide dismutase (SOD) using a commercial assay kit purchased from Cyman Chemical (Ann Arbor, MI, USA) according to the manufacturer's instructions.

2.6. Enzyme-Linked Immunosorbent Assays. Culture supernatants from the control and cocultured hVFFs were examined for levels of interleukin- (IL-) $1 \beta$ and tumor necrosis factor-alpha (TNF- $\alpha$ ) using human ELISA kits (eBioscience, San Diego, CA, USA, and ThermoFisher, Waltham, MA, USA), according to the manufacturer's instructions.

2.7. Quantitative Real-Time PCR (qRT-PCR). Total RNA from hVFFs was isolated using a TRIzol Reagent (ThermoFisher) according to the manufacturer's protocol and was then treated with RNase-free DNase to eliminate genomic DNA contamination. Total RNA $(2 \mu \mathrm{g})$ was reverse transcribed into cDNA with AccuPower ${ }^{\circledR}$ CycleScript RT PreMix (Bioneer, Daejeon, Republic of Korea). Real-time quantitative reverse transcription polymerase chain reaction (qRT-PCR) was performed on an ABI PRISM 7500 Real-Time PCR System using AccuPower ${ }^{\circledR} 2 \mathrm{X}$ GreenStar ${ }^{\mathrm{TM}}$ qPCR Master Mix (Bioneer). Reaction conditions were $10 \mathrm{~min}$ at $95^{\circ} \mathrm{C}$ (one cycle), $10 \mathrm{sec}$ at $95^{\circ} \mathrm{C}$, and $30 \mathrm{sec}$ at $60^{\circ} \mathrm{C}$ (40 cycles). Normalization consisted of using the differences between the cycle thresholds (delta CT) and the expression level for GAPDH to calculate the delta CT/target gene delta CT ratio. Delta/delta CT corresponds to the differences between the delta CT and the internal control gene. The information on primer sequences is shown in Table 1.
2.8. Immunofluorescent Staining. Cells were washed in PBS and fixed on coverslips with 3\% paraformaldehyde (Biosesang, Bundang, Korea) for $10 \mathrm{~min}$ at room temperature and rinsed twice with PBS. Cells were permeabilized with 0.25\% Triton X-100 (Roche Molecular Biochemicals, Indianapolis, IN) in PBS for $10 \mathrm{~min}$ at room temperature and then blocked with $1 \%$ bovine serum albumin (Sigma) for $1 \mathrm{~h}$.After blocking, cells were incubated with primary antibodies for collagen I (Abcam, ab34710), collagen III (Abcam, ab7778) and collagen IV (Abcam, ab6586) at $4^{\circ} \mathrm{C}$ overnight and then washed with PBS $(3 \times 5 \mathrm{~min})$.Cells were incubated with FITC- or TRICT-conjugated goat antimouse antibodies (Molecular Probes, Eugene, OR) (1:1000)for $1 \mathrm{~h}$ at room temperature, followed by the washing with PBS $(3 \times 5 \mathrm{~min})$. Nuclei were stained with $0.1 \mu \mathrm{g} / \mathrm{mL}$ DAPI. The coverslips were mounted face down on microscope slides with mounting medium (Vector Laboratories, Inc., Burlingame, CA) to be viewed on a confocal microscope (Leica DM4000/600M, Versatile Upright Microscope for Materials Analysis).

2.9. Matrix Metalloproteinase (MMP) Activity Assay. The MMP activity assay kit (Abcam, Cambridge, MA, USA) was used to analyze the activity of MMPs from cell supernatants by incubating with a fluorescent substrate (fluorescence resonance energy transfer (FRET) peptide). In the intact FRET peptide, the fluorescence of one part is silenced by another. After cleavage into two separate fragments by MMPs, the fluorescence is recovered. The probe signal was then detected using a fluorescence microplate reader at $\mathrm{Ex} / \mathrm{Em}=490 / 525 \mathrm{~nm}\left(\mathrm{Gen} 5^{\mathrm{TM}}\right.$ Microplate Reader, VT, USA).

2.10. Statistical Analysis. Data analysis was performed using SigmaPlot 10.0 (Systat Software Inc., San Jose, CA, USA). All experimental data were expressed as the mean \pm SD. Differences between the groups were analyzed using Student's $t$-test and one-way analysis of variance (ANOVA) followed by Bonferroni post hoc test for multigroup comparisons with $P<0.05$ considered as statistically significant.

\section{Results}

3.1. Cell Viability and Proliferation of hVFFs Were Decreased after Exogenous Induction of Oxidative Stress and Partially Restored by TMSC Coculture. To investigate whether hVFF proliferation is affected following induction of oxidative stress, cell proliferation and viability were performed after various concentration of tBHP treatment. We found that cell viability was significantly decreased in a dose-dependent manner following treatment with tBHP (Figure 1(a)). To establish optimal experimental conditions for assessing the effects of oxidative stress in hVFFs, $300 \mu \mathrm{M}$ of tBHP treatment was selected for further protocols since it elicited the lowest level of cytotoxicity in the viability assay (Figure 1(a)). Then, we examined the antioxidant capacity of TMSCs on tBHP-treated hVFFs by culturing hVFFs with TMSCs. We observed that TMSCs partially restored cell proliferation in a dose-dependent manner. The coculture ratio 
TABle 1: Primer sequences.

\begin{tabular}{lcr}
\hline Gene & Primer & Sequence \\
\hline Human HO1 & Forward & $5^{\prime}$-GAGGGAAGCCCCCACTCAAC-3' \\
Human SOD1 & Reverse & $5^{\prime}$-GGCATAAAGCCCTACAGCAACT-3' $^{\prime}$ \\
Human SOD2 & Forward & $5^{\prime}$-ACAAAGATGGTGTGGCCGAT-3' \\
& Reverse & $5^{\prime}$-AACGACTTCCAGCGTTTCCT-3' \\
Human GAPDH & Forward & $5^{\prime}$-GGCCTACGTGAACAACCTGA-3' \\
Human collagen I & Reverse & $5^{\prime}$-GTTCTCCACCACCGTTAGGG-3' \\
& Forward & $5^{\prime}$-GCGACCTGGAAGTCCAACTA-3' \\
Human collagen III & Reverse & $5^{\prime}$-ATCTGCTGCATCTGCTTGG-3' \\
& Forward & $5^{\prime}$-CCTCAAGAGAAGGCTCACGATGGTG-3' \\
Human collagen IV & Reverse & $5^{\prime}$-AGGTCTCACCAGTCTCCATGTTGCA-3' $^{\prime}$ \\
& Forward & $5^{\prime}$-GCTCTGCTTCATCCCACTATTA-3' \\
\hline
\end{tabular}

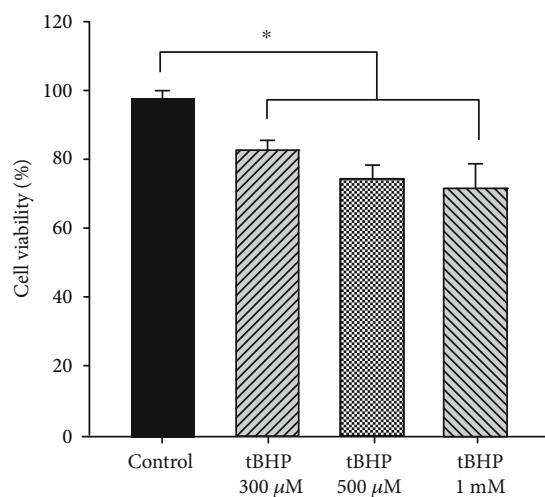

(a)
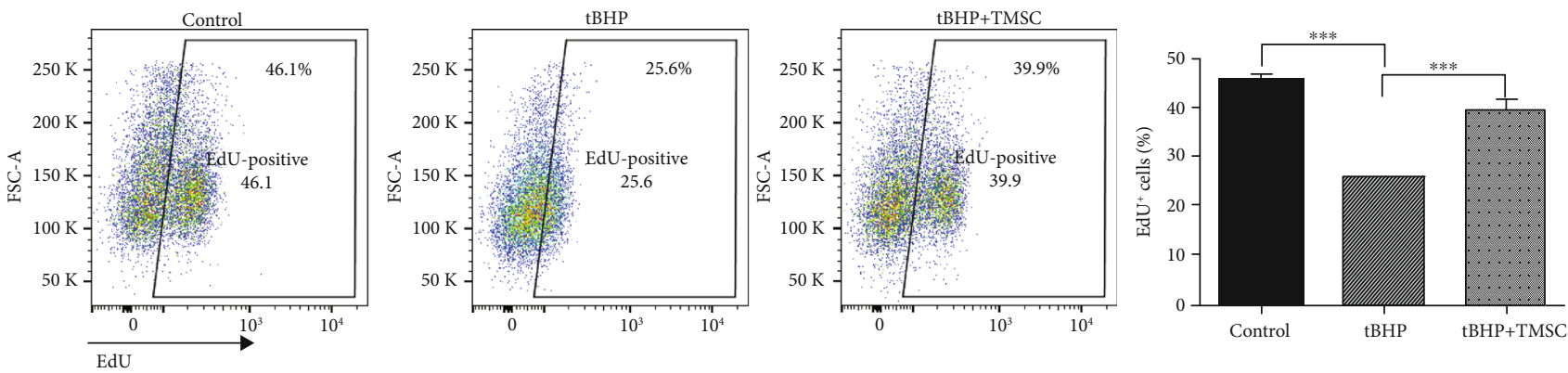

(c)

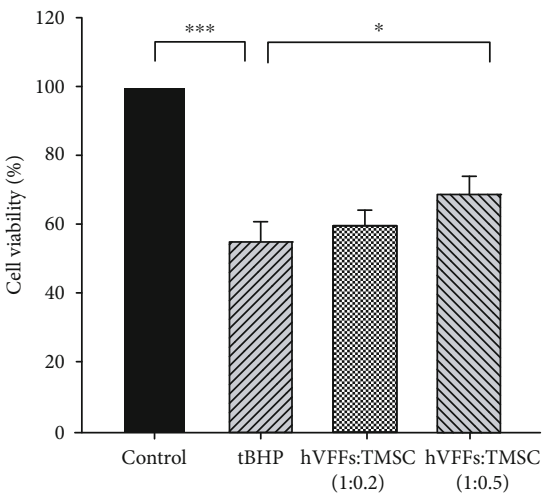

(b)

FIGURE 1: Viability and proliferation of human vocal fold fibroblasts (hVFFs) after tert-butyl hydroperoxide (tBHP) treatment and coculture with tonsil-derived mesenchymal stem cells (TMSCs). (a) Cell viability was significantly decreased following treatment with tBHP in a dose-dependent manner. (b) Of the different hVFF:TMSC ratios examined, 1:0.5 ratio exhibited significant recovery of cell viability. (c) Representative flow cytometry results and integrated proportion for EdU ${ }^{+}$proliferating cells. Results are 3 technical replicates of TMSC from one donor. Representative results from two different TMSCs with similar tendency were presented. For multigroup comparison, ANOVA followed by the Bonferroni post hoc test was used for statistics. Results are shown as the mean \pm SD. ${ }^{*} P<0.05,{ }^{* * *} P<0.001$. 


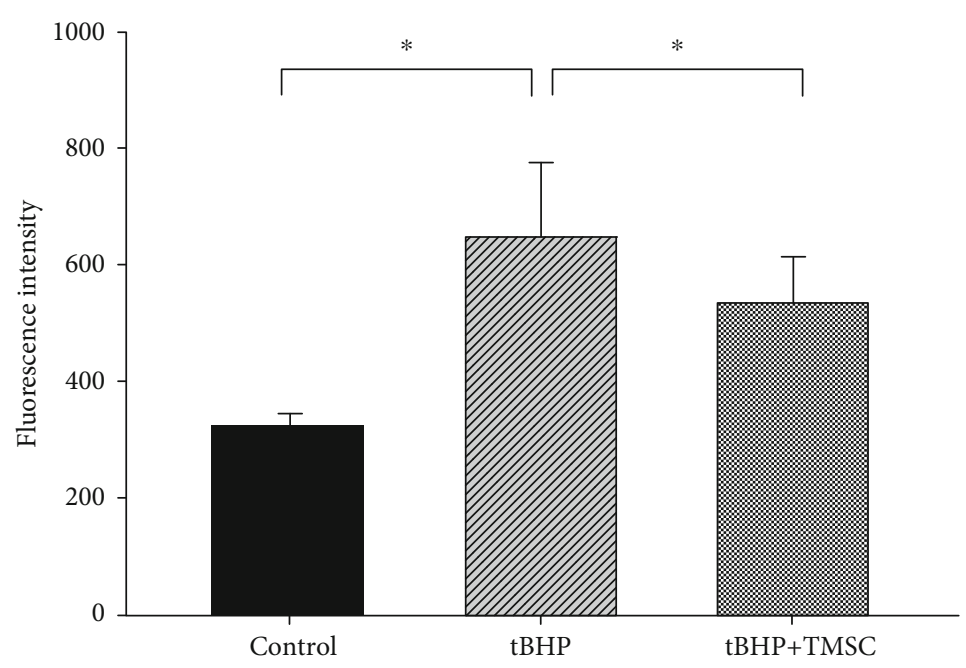

(a)

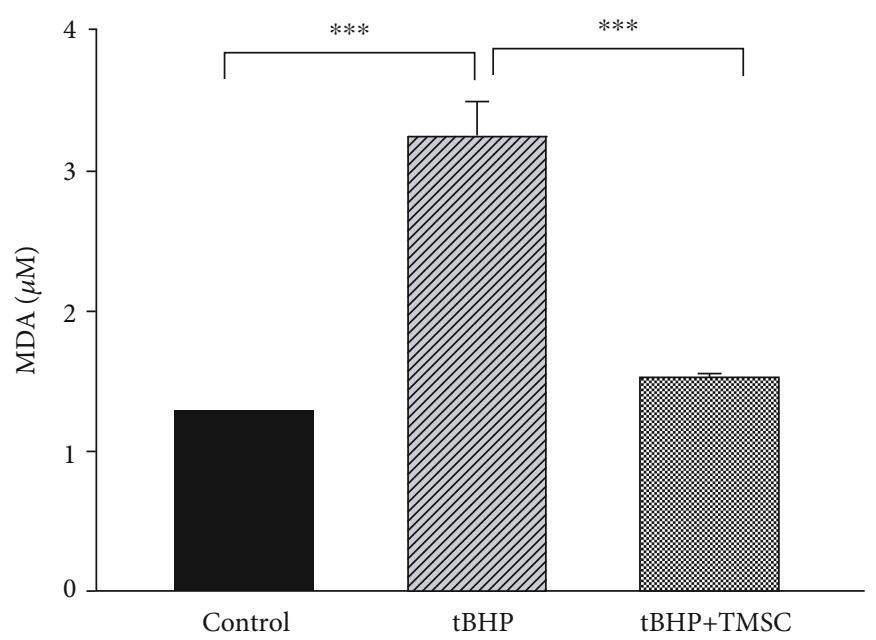

(b)

FIGURE 2: Analysis of ROS production and expression of MDA following treatment with tBHP. (a) The fluorescence intensity in the DCFDA array increased following treatment of hVFFs with tBHP. After coculturing with TMSC, tBHP-treated hVFFs exhibited significantly decreased fluorescence intensity levels. (b) MDA was significantly increased in tBHP-treated hVFFs, while coculture with TMSCs significantly decreased MDA levels. Results are 3 technical replicates of TMSC from one donor. Representative results from two different TMSCs with similar tendency were presented. For multigroup comparison, ANOVA followed by the Bonferroni post hoc test was used for statistics. Results are shown as the mean \pm SD. ${ }^{*} P<0.05,{ }^{* * *} P<0.001$.

(hVFFs:TMSCs) of $1: 0.5$ was selected for the subsequent analyses because it demonstrated significant protective effects for cell viability (Figure 1(b)). We next investigated the impact of tBHP and TMSCs on proliferative capacity of hVFFs in an established setting using the EdU incorporation assay. In line with CCK-8 assay results, $300 \mu \mathrm{M}$ of tBHP significantly reduced the cell proliferation rate in exposed cells, while coculture with TMSCs could rescue the phenomenon. The $\mathrm{EdU}^{+}$cell proportion was declined upon $\mathrm{tBHP}$ treatment from $46 \%$ to $26 \%$ and further restored by TMSC coculture up to $40 \%$ (Figure $1(\mathrm{c})$ ).

3.2. TMSCs Reduced ROS Production in hVFFs Induced by tBHP Treatment. To determine the level of ROS production in hVFFs following induction of oxidative stress as well as the regulation of ROS production by TMSCs, DCFDA arrays were performed. Our results reveal that the level of ROS was significantly increased in hVFFs after tBHP treatment. However, coculturing with TMSC effectively inhibited the tBHP-mediated ROS production (Figure 2(a)). Further, the expression of intracellular MDA was found to be significantly increased in tBHP-treated hVFFs; however, coculturing with TMSC served to significantly reduce intracellular levels of MDA (Figure 2(b)).

3.3. TMSCs Partially Restored the Production of Antioxidant Enzymes in hVFFs Exposed to Oxidative Stress. We next investigated whether the endogenous activity of antioxidant enzymes in hVFFs was affected by tBHP and TMSCs could restore this change. We found that while the activity of superoxide dismutase (SOD) and catalase and the expression of glutathione peroxidase (GPX) and glutathione (GSH) were 


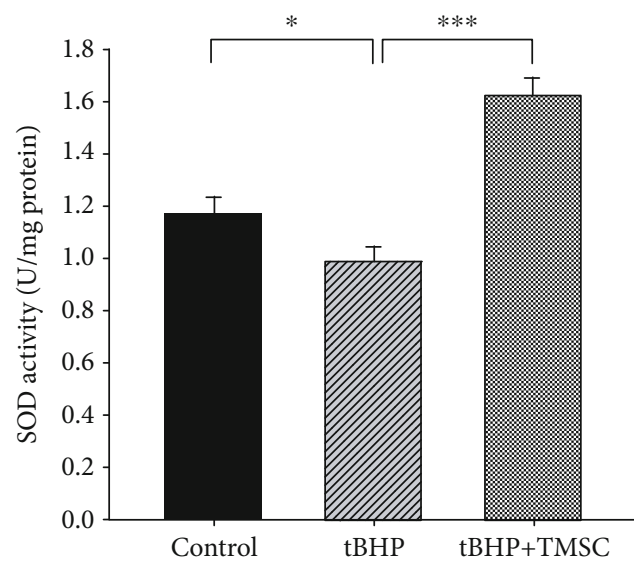

(a)

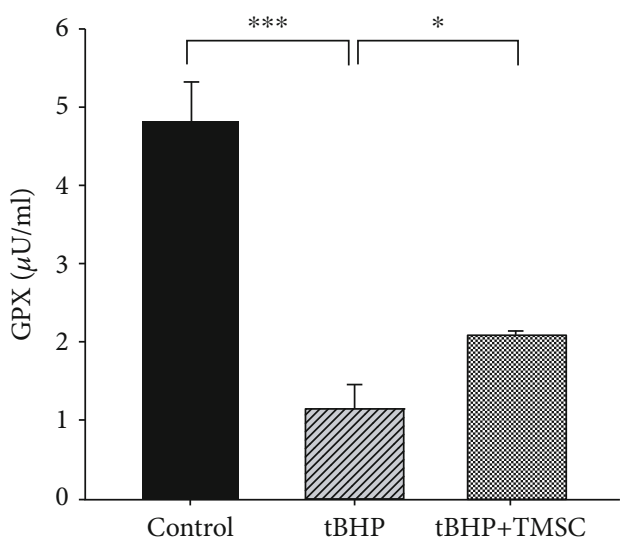

(c)

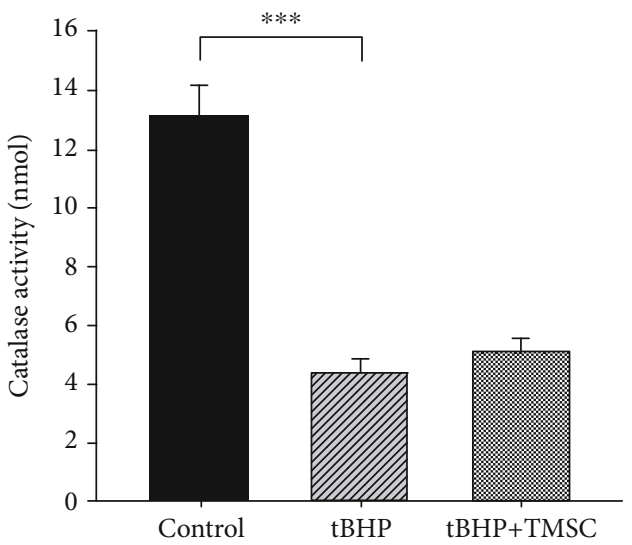

(b)

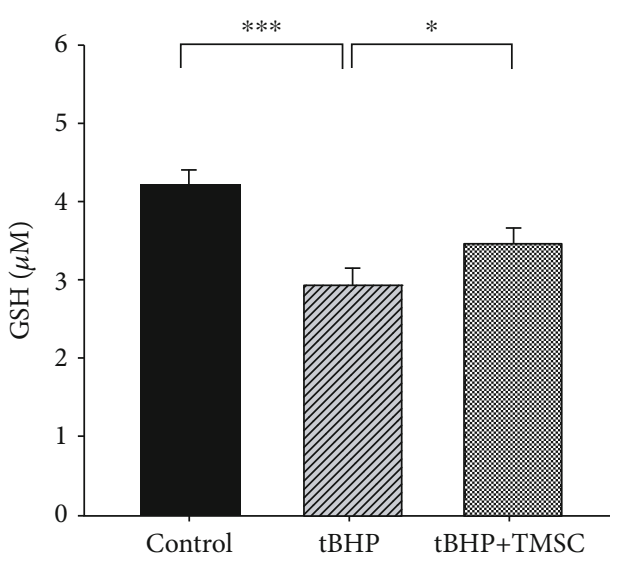

(d)

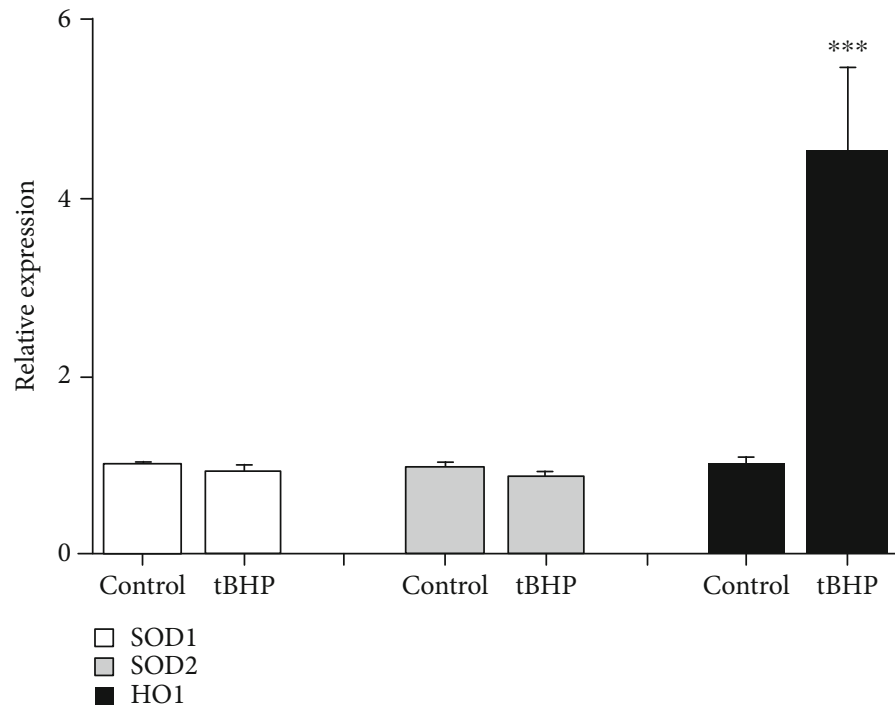

(e)

Figure 3: The dynamic changes in activity and expression of malondialdehyde (MDA) and other antioxidant enzymes. (a-d) The activity and expression of various antioxidant enzymes including superoxide dismutase (SOD), catalase, glutathione peroxidase (GPX), and glutathione (GSH) in hVFFs were significantly increased following treatment with tBHP and partially restored after coculture with TMSC. (e) qRTPCR results showing that the HO1 mRNA level in TMSCs was upregulated upon tBHP treatment. No significant change in SOD1 and SOD2 transcript levels was observed. Results are 3 technical replicates of TMSC from one donor. Representative results from two different TMSCs with similar tendency were presented. For multigroup comparison, ANOVA followed by the Bonferroni post hoc test was used for statistics. Results are shown as the mean \pm SD. ${ }^{*} P<0.05$ and ${ }^{* * *} P<0.001$. 

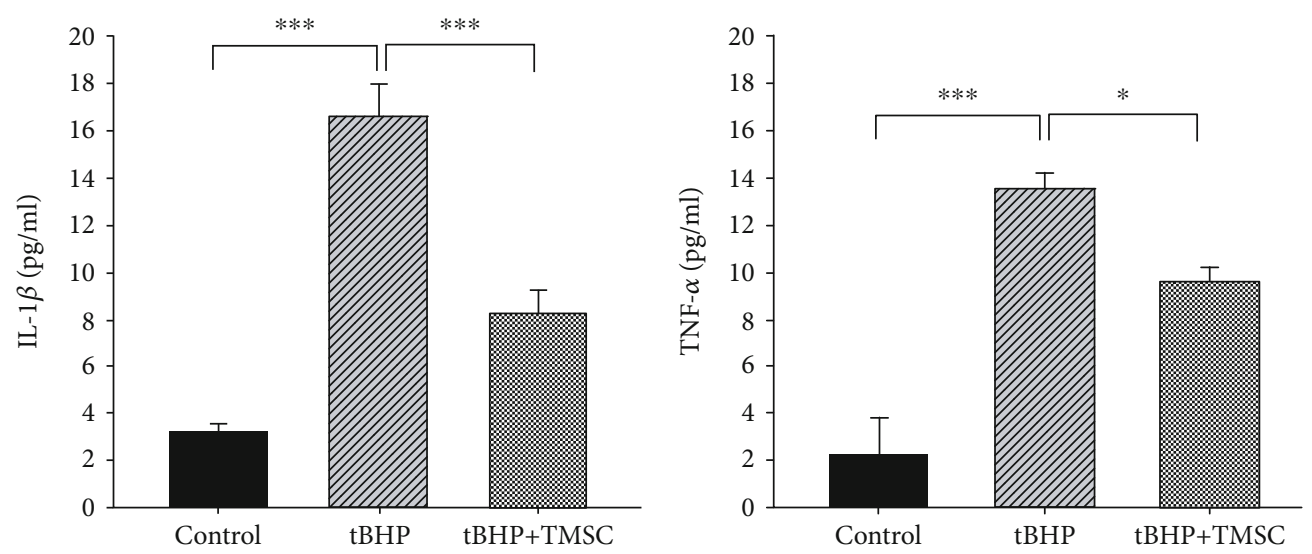

FIgURE 4: The dynamic changes in expression of inflammatory cytokines during oxidative stress responses. The expression of the representative inflammatory cytokines, IL- $1 \beta$ and TNF- $\alpha$, was found to be significantly upregulated in tBHP-treated hVFFs yet downregulated following coculture with TMSC. Results are 3 technical replicates of TMSC from one donor. Representative results from two different TMSCs with similar tendency were presented. For multigroup comparison, ANOVA followed by the Bonferroni post hoc test was used for statistics. Results are shown as the mean \pm SD. ${ }^{*} P<0.05,{ }^{* * *}<0.001$.

significantly decreased after tBHP treatment, coculturing of tBHP-treated hVFFs with TMSCs resulted in partial restoration (Figures 3(a)-3(d)). Since oxidative stress can also influence the antioxidant capacity of TMSCs by regulating the production of related enzymes, we treated tBHP to TMSCs and evaluated the transcriptome levels of reported antioxidant enzymes in TMSCs using qRT-PCR. It is revealed that tBHP could induce an approximately 4.5 -fold increase in heme oxygenase1 (HO1) gene transcription in TMSCs compared with the control, implying that antioxidative function of TMSCs can be enhanced under oxidative stress condition (Figure 3(e)).

3.4. TMSCs Reduce the Production of Inflammatory Cytokines in hVFFs Exposed to Oxidative Stress. To determine whether the inflammatory response is affected by oxidative stress, we quantified the level of representative inflammatory cytokines from hVFFs after treatment with tBHP in the presence or absence of TMSCs. Our results show that within the culture supernatant, the concentration of IL- $1 \beta$ and TNF- $\alpha$ was significantly increased in tBHP-treated hVFFs yet was decreased in TMSC cocultured cells (Figure 4).

3.5. TMSCs Partially Counteracts the Inhibitory Effect Induced by Oxidative Stress on the Expression and Activity of Extracellular Matrix and Matrix Metalloproteinase. Since abnormal accumulation of ECM often leads to pathologic damages and fibrosis, we next explored the endogenous ECM production level in hVFFs after induction of oxidative stress. We first evaluated the expression changes in various collagen subtypes using qRT-PCR. We found that the relative mRNA expression of collagens I, III, and IV was significantly upregulated following treatment with tBHP. Of interest, coculture with TMSCs resulted in partial reduction in the expression of all collagen subtypes (Figures 5(a)-5(c)). These phenomena were confirmed by immunocytochemistry showing that fluorescence intensity of collagen proteins in hVFFs was increased by tBHP treatment, while TMSCs could partially reverse the upregulation of collagens (Figures 5(d) and 5(e)). To investigate the underlying mechanisms for the ECM reduction, the activity of MMPs, major responsible enzymes for ECM degradation, was determined in culture supernatant of hVFFs collected from designated experimental settings. It is noted that oxidative stress induced by tBHP treatment seemed to inhibit MMP activity of hVFFs (Figure 5(f)). We also found clear evidence of TMSC protection against oxidative stress in terms of MMP activity restoration; indeed, hVFFs cocultured with TMSCs for 24 hours before tBHP treatment could maintain a higher level of MMP activity, leading to decreased accumulation of collagens compared with controls. The results from the additional MMP activity assay revealed that treatment with tBHP slightly suppressed the activity of MMP 1 compared to the control and strongly inhibited those of MMPs 8, 2, and 9 (Figures 6(a)-6(d)). Interestingly, the activities of all MMPs were partially restored following coculture with TMSCs (Figures 6(a)-6(d)).

\section{Discussion}

Oxidative stress is involved in various diseases such as cancer, inflammatory conditions, neurodegenerative diseases, aging, and diabetes. In the field of laryngology, studies have reported on the relationship between oxidative stress and vocal fold scarring as well as aging vocal folds. Specifically, ROS have been shown to be significantly upregulated during the early stage of vocal fold wound healing [6]. Alternatively, treatment with astaxanthin, a strong antioxidant, induced significant upregulation in the expression of procollagen and fibroblast growth factor (FGF) within the lamina propria on the first day after injury in a rat model of vocal fold injury [21]. These results suggest that astaxanthin may function to prevent vocal fold scarring by regulating oxidative stress during the early phase of vocal fold wound healing [21]. 


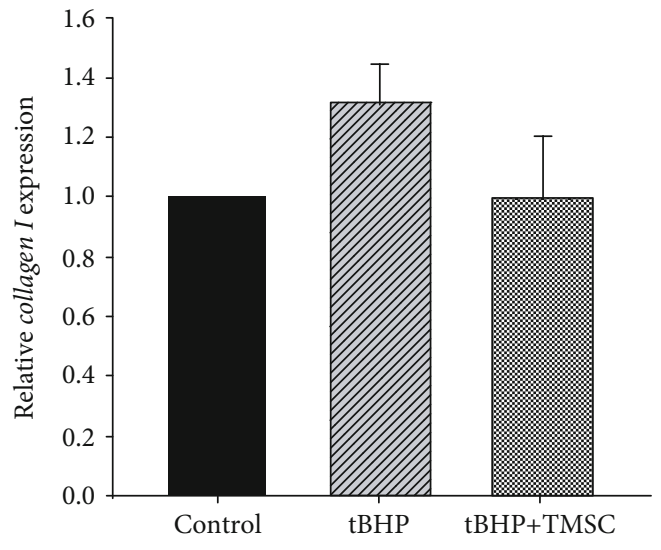

(a)

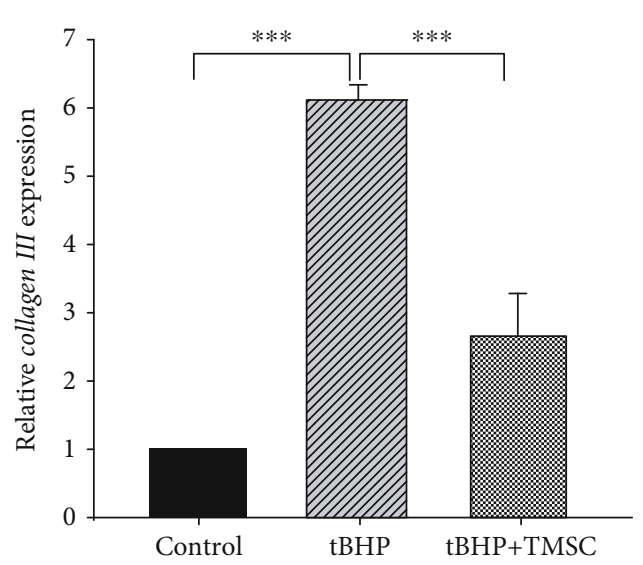

(b)
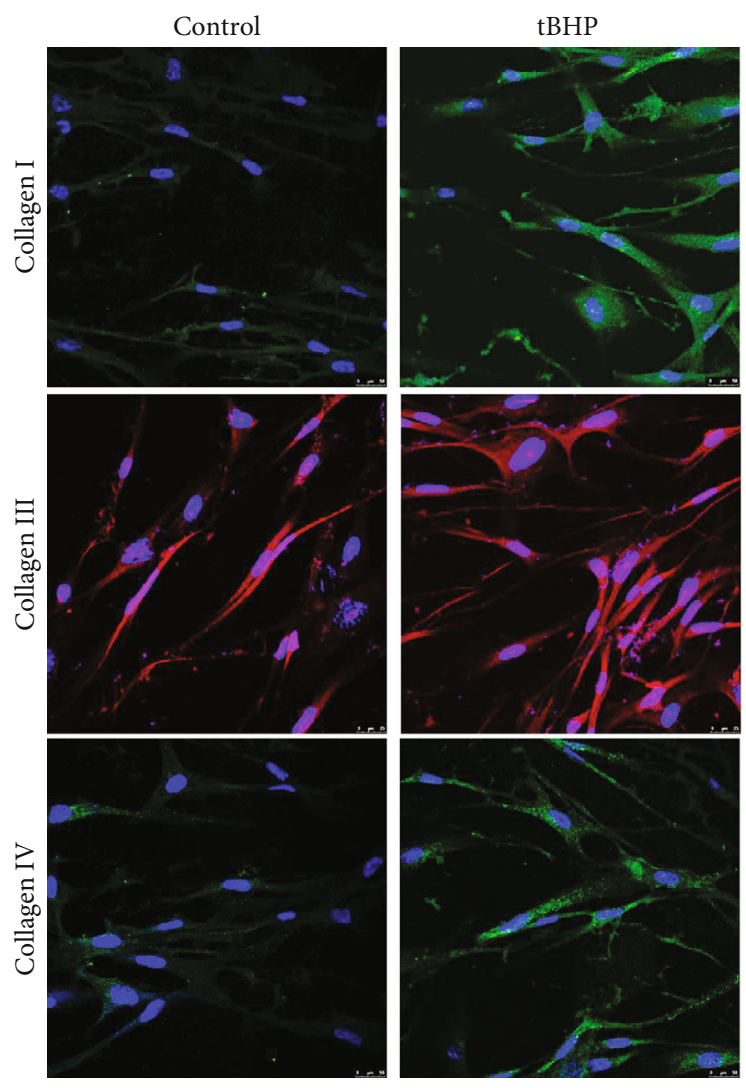

(d)

tBHP+TMSC

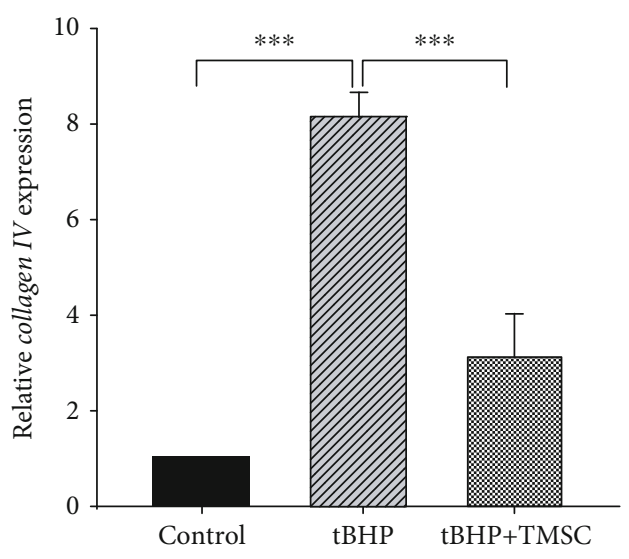

(c)

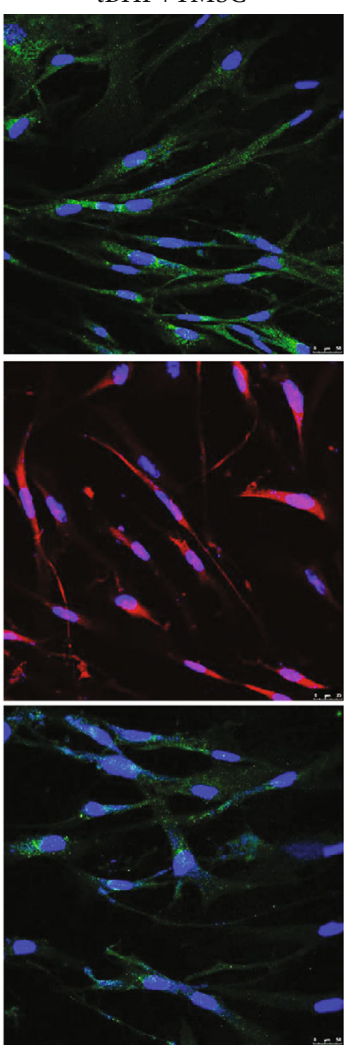

Figure 5: Continued. 


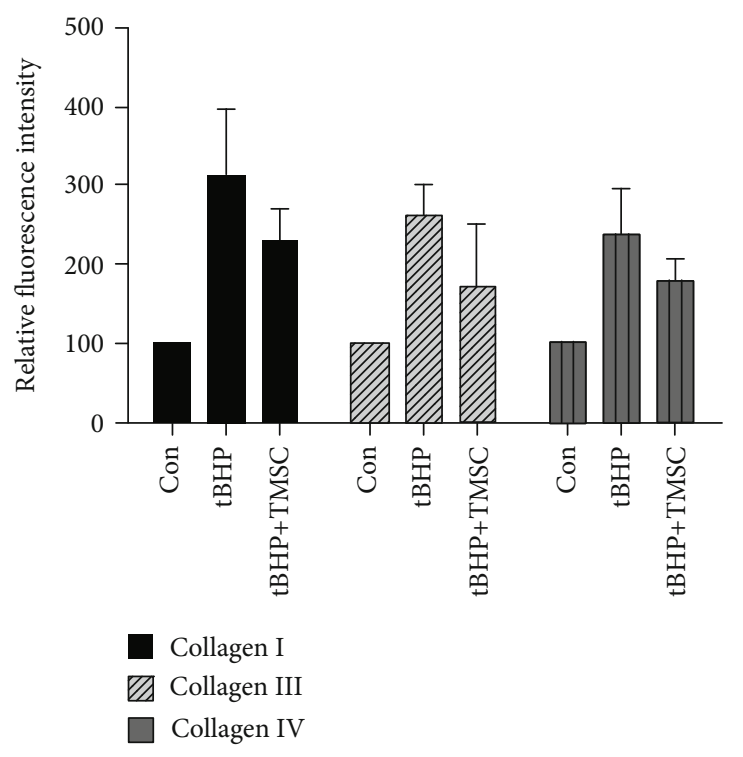

(e)

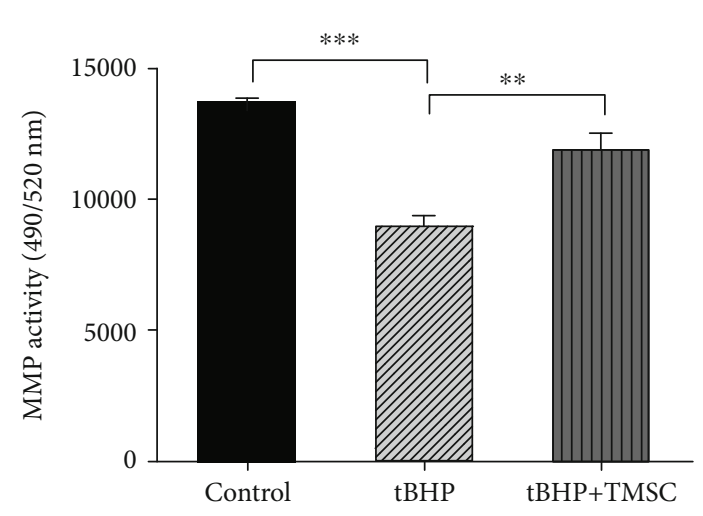

(f)

FIgURE 5: Dynamic changes in expression of extracellular matrix-(ECM-) related genes. (a-c) Relative mRNA expression of collagens I, III, and IV was determined to be significantly upregulated following tBHP treatment. Coculturing with TMSCs served to downregulate the expression of all collagen subtypes. (d, e) The representative immunofluorescence images for collagen staining (d). Images were quantified by measuring fluorescence intensity (e). Similar to qRT-PCR results, oxidative stress enhanced collagen expression in hVFFs while TMSC counteracted tBHP's impact. (f) MMP activity assay results showing that decreased MMP activity in tBHP-treated hVFFs was significantly restored after coculturing with TMSCs. Results are 3 technical replicates of TMSC from one donor. Representative results from two different TMSCs with similar tendency were presented. For multigroup comparison, ANOVA followed by the Bonferroni post hoc test was used for statistics. Results are shown as the mean \pm SD. ${ }^{*} P<0.05,{ }^{* *} P<0.01$, and ${ }^{* * *} P<0.001$.

Oxidative stress has also been found to be associated with aging in many tissues, and thus, aging of vocal folds, which causes dysphonia, may also be associated with ROS production. Further, the administration of astaxanthin may serve to attenuate the age-associated reduction in hyaluronic acid (HA) deposition in the lamina propria and the expression of HGF and bFGF [22]. Moreover, Mizuta et al. suggested that astaxanthin has the potential to prevent age-associated changes in human voices [22]. Smoking is also a welldescribed etiological factor for both Reinke's edema and laryngeal cancer. Branski et al. reported that cigarette smoke condensate caused an increase in intracellular ROS within hVFFs, while decreasing the proliferative capacity and migration of these cells in a dose-dependent manner [23]. Thus, it was postulated that ROS generation contributed to the pathogenesis associated with Reinke's edema.

Oxidative stress has been described as a contributing factor to the pathogenesis of various laryngeal conditions including vocal fold scarring, aging vocal folds, and Reinke's edema. Excessive accumulation of ROS and other free radicals can induce oxidative stress in tissues. Accumulation of intracellular ROS binds to lipids and proteins and reduces the viability of cells. In this study, oxidative stress induced by tBHP also increased the production of ROS and MDA in hVFFs. In addition, tBHP caused a decrease in the expression of antioxidative molecules, namely, SOD, catalase, GPX, and GSH, and reduced the viability of hVFFs. However, coculturing with TMSCs acted to decrease produc- tion of ROS and MDA, while increasing expression of antioxidative enzymes and improving the viability of hVFFs. These findings suggest that TMSCs exhibit cytoprotective effects against oxidative stress, which agree with results from previous studies that reported bone marrow stromal cells (BMSCs) exhibiting increased expression of antioxidant molecules and resistance to ROS [24].

Oxidative stress is strongly associated with inflammation primarily caused by the activation of oxidative radicals which stimulate production of inflammatory chemokines, such as TNF- $\alpha$, IL-1 $\beta$, IL-6, and IL-8 [25]. Increased expression of inflammatory chemokines induces changes in ECM constituents and increases tissue fibrosis. In the current study, treatment with tBHP resulted in increased secretion of TNF- $\alpha$ and IL-1, increased expression of collagens III and IV, and decreased activity of MMPs 1, 2, 8, and 9. However, coculturing with TMSCs served to downregulate the expression of TNF- $\alpha$ and IL-1, as well as of collagens III and IV. Moreover, the activity of MMPs 1,2,8, and 9 increased when cocultured with TMSCs prior to tBHP treatment. These findings suggest that oxidative stress increases the secretion of inflammatory cytokines and negatively affects the production and degradation of different ECM components and thus may be the cause of the observed changes within the ECM in vocal fold scarring and aging vocal folds. Alternatively, TMSCs function to inhibit the secretion of inflammatory cytokines and counteract the oxidative stress-induced effects on ECM component degradation and production. 


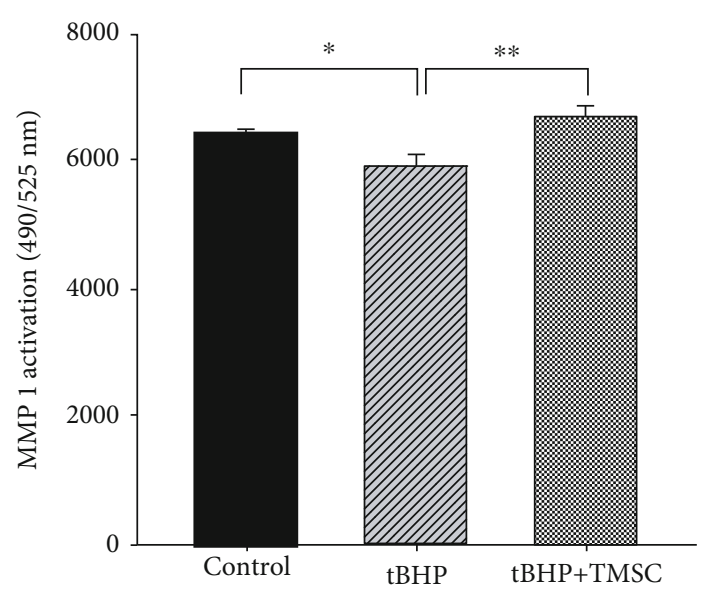

(a)

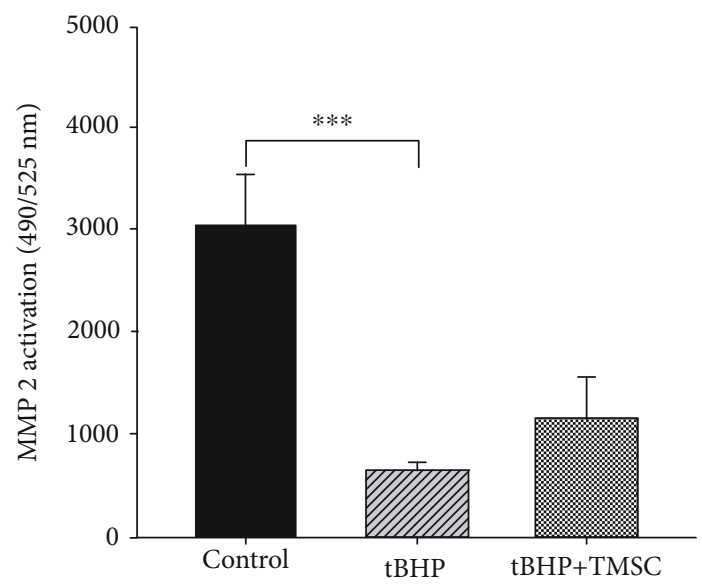

(c)

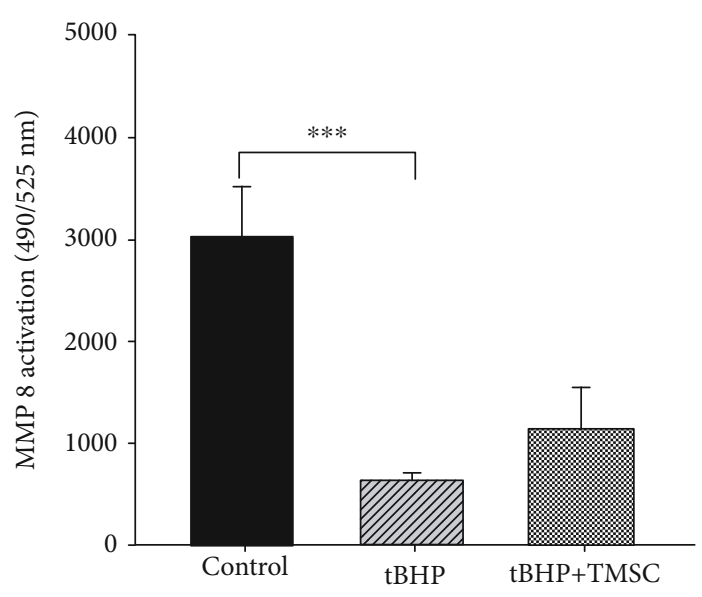

(b)

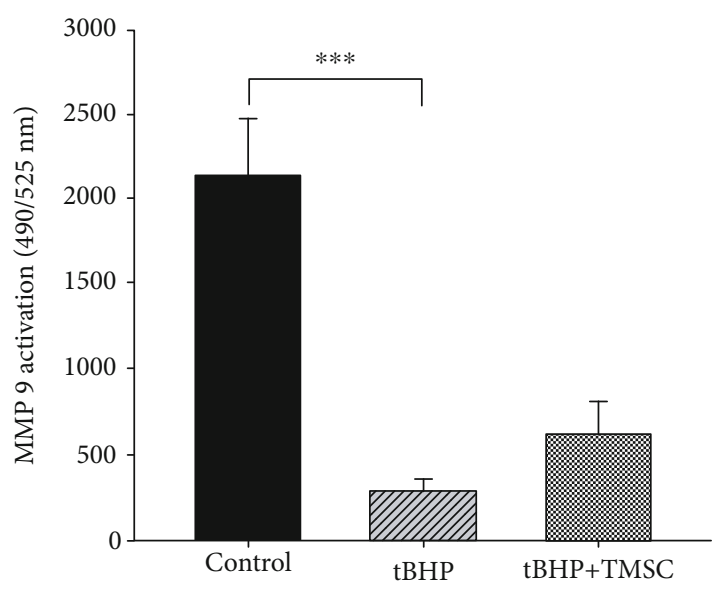

(d)

FIgURE 6: Matrix metalloproteinase (MMP) activity assay. (a-d) Treatment with tBHP slightly suppressed the activity of MMP 1 and strongly inhibited those of MMPs 8, 2, and 9. The activities of all MMPs were significantly restored after coculturing with TMSCs. Results are 3 technical replicates of TMSC from one donor. Representative results from two different TMSCs with similar tendency were presented. For multigroup comparison, ANOVA followed by the Bonferroni post hoc test was used for statistics. Results are shown as the mean \pm SD. ${ }^{*} P<0.05,{ }^{* *} P<0.01,{ }^{* * *} P<0.001$.

Specific ECM-related genes have been found to be differentially expressed in vocal fold scars of rats. Specifically, both collagen I and fibronectin have been found to be upregulated and HA downregulated [26]. Similarly, within aging vocal folds, the changes in ECM within the lamina propria layer have been associated with increased levels of collagen I, collagen III, MMP 1, and MMP 8 and decreased HA and elastin $[27,28]$. Similarly, within our study, we have shown that the proliferative capacity of hVFFs and the expression of ECM-related genes were negatively impacted following exposure to oxidative stress. Specifically, the expression of collagens I, III, and IV was increased, while elastin remained unchanged. Although these changes in ECM-related genes within hVFFs were not ultimately consistent with changes observed in ECM of actual vocal fold scars or aging, similarities were observed, suggesting that oxidative stress may contribute to development of changes within ECM in aging vocal folds or vocal fold scarring. One might envision that this ECM-regulating ability of TMSCs might be beneficial for the wound healing process. In our previous study, we demonstrated regenerative potential of TMSCs in wound healing through the regulation on inflammation, proliferation, and remodeling of various skin cells [14]. In the study, TMSCs enhanced proliferation of skin fibroblasts and keratinocytes while suppressing the differentiation of fibroblasts into myofibroblasts. In the current study, MDA was found to be increased following exposure to oxidative stress, and TMSCs served to increase the proliferative capacity of hVFFs and collagen I expression in fibroblasts. These findings suggest that TMSCs improve wound healing by functioning as an effective antioxidant agent. These results are consistent with other studies that have reported an antioxidant function for MSC [16, 17, 29]. Specifically, Cui et al. reported that bone marrow MSCs ameliorate oxidative stress, inhibit inflammation, and promote secretion of neurotrophins thereby providing protective effects for retina ganglion cell-5, by suppressing $\mathrm{H}_{2} \mathrm{O}_{2}$-induced cell damage [29]. Furthermore, Wang et al. reported that human amnion-derived MSCs exhibit a strong 
potential for treating oxidative stress-mediated bone deficiency via activation of the ERK1/2 MAPK signal transduction pathway [17]. We have also shown that TMSCs elicit an antioxidant effect that serves to counteract the oxidative stress-induced reduction in hVFF proliferation and changes in the expression of ECM-related genes. The antioxidant function of TMSC is postulated to be associated with improved wound healing and preventing accumulation of scar tissue.

\section{Conclusions}

Oxidative stress caused by tBHP increases inflammatory cytokines, reduces expression of antioxidant enzymes, and induces changes in ECM components in hVFFs. These changes are consistent with the phenotypes observed in both aging vocal folds and vocal fold scarring. However, TMSCs exhibit a strong antioxidant capacity that counteracts the effects induced by oxidative stress in hVFFs. Further research is needed to identify genes, miRNAs, or proteins that are associated with the observed antioxidant and antiaging function of TMSCs. This research would, therefore, inform the development of effective technologies in the field of regenerative medicine.

\section{Data Availability}

The data used to support the findings of this study are included within the article.

\section{Conflicts of Interest}

The authors declare no conflicts of interest.

\section{Authors' Contributions}

Sung-Chan Shin and Hyung-Sik Kim contributed equally to this work.

\section{Acknowledgments}

This work was supported by the National Research Foundation of Korea (NRF) grant funded by the Korea government (MSIT) (No. NRF-2017R1E1A1A01074316, NRF2016R1D1A3B01015539, and NRF-2019R1A2C2085876).

\section{Supplementary Materials}

Characterization of human tonsil-derived mesenchymal stem cells (TMSCs). (Supplementary Materials)

\section{References}

[1] J. P. Kehrer, T. E. Tipple, J. D. Robertson, and C. V. Smith, "Free radicals as mediators of tissue injury and disease," Reference Module in Biomedical Sciences, pp. 1040-8444, 2015.

[2] R. A. Floyd, R. A. Towner, T. He, K. Hensley, and K. R. Maples, "Translational research involving oxidative stress and diseases of aging," Free Radical Biology \& Medicine, vol. 51, no. 5, pp. 931-941, 2011.
[3] S. Roy, S. Khanna, K. Nallu, T. K. Hunt, and C. K. Sen, "Dermal wound healing is subject to redox control," Molecular Therapy, vol. 13, no. 1, pp. 211-220, 2006.

[4] C. K. Sen and S. Roy, "Redox signals in wound healing," Biochimica et Biophysica Acta, vol. 1780, no. 11, pp. 1348-1361, 2008.

[5] M. Wlaschek and K. Scharffetter-Kochanek, "Oxidative stress in chronic venous leg ulcers," Wound Repair and Regeneration, vol. 13, no. 5, pp. 452-461, 2005.

[6] M. Mizuta, S. Hirano, S. Ohno et al., "Expression of reactive oxygen species during wound healing of vocal folds in a rat model," The Annals of Otology, Rhinology, and Laryngology, vol. 121, no. 12, pp. 804-810, 2012.

[7] M. Mizuta, S. Hirano, S. Ohno, S. Kanemaru, T. Nakamura, and J. Ito, "Restoration of scarred vocal folds using 5 amino acid-deleted type hepatocyte growth factor," Laryngoscope, vol. 124, no. 3, pp. E81-E86, 2014.

[8] M. J. Ban, J. H. Park, J. W. Kim et al., "The efficacy of fibroblast growth factor for the treatment of chronic vocal fold scarring: from animal model to clinical application," Clinical and Experimental Otorhinolaryngology, vol. 10, no. 4, pp. 349-356, 2017.

[9] S. Hirano, D. M. Bless, B. Rousseau et al., "Prevention of vocal fold scarring by topical injection of hepatocyte growth factor in a rabbit model," The Laryngoscope, vol. 114, no. 3, pp. 548-556, 2004.

[10] S. Ohno, S. Hirano, S. I. Kanemaru et al., "Transforming growth factor $\beta 3$ for the prevention of vocal fold scarring," Laryngoscope, vol. 122, no. 3, pp. 583-589, 2012.

[11] B. J. Lee, S. G. Wang, J. C. Lee et al., "The prevention of vocal fold scarring using autologous adipose tissue-derived stromal cells," Cells, Tissues, Organs, vol. 184, no. 3-4, pp. 198-204, 2006.

[12] A. Mattei, J. Magalon, B. Bertrand, C. Philandrianos, J. Veran, and A. Giovanni, "Cell therapy and vocal fold scarring," European Annals of Otorhinolaryngology, Head and Neck Diseases, vol. 134, no. 5, pp. 339-345, 2017.

[13] R. Luo, Y. Lu, J. Liu, J. Cheng, and Y. Chen, "Enhancement of the efficacy of mesenchymal stem cells in the treatment of ischemic diseases," Biomedicine \& Pharmacotherapy, vol. 109, pp. 2022-2034, 2019.

[14] S. C. Shin, Y. Seo, H. Y. Park et al., "Regenerative potential of tonsil mesenchymal stem cells on surgical cutaneous defect," Cell Death \& Disease, vol. 9, no. 2, p. 183, 2018.

[15] G. Camussi, M. C. Deregibus, and V. Cantaluppi, "Role of stem-cell-derived microvesicles in the paracrine action of stem cells," Biochemical Society Transactions, vol. 41, no. 1, pp. 283287, 2013.

[16] A. Monsel, Y. G. Zhu, S. Gennai, Q. Hao, J. Liu, and J. W. Lee, "Cell-based therapy for acute organ injury: preclinical evidence and ongoing clinical trials using mesenchymal stem cells," Anesthesiology, vol. 121, no. 5, pp. 1099-1121, 2014.

[17] Y. Wang, J. Ma, Y. Du, J. Miao, and N. Chen, "Human amnion-derived mesenchymal stem cells protect human bone marrow mesenchymal stem cells against oxidative stressmediated dysfunction via ERK1/2 MAPK signaling," Molecules and Cells, vol. 39, no. 3, pp. 186-194, 2016.

[18] B. J. Lee, D. W. Kang, H. Y. Park et al., "Isolation and localization of mesenchymal stem cells in human palatine tonsil by W5C5 (SUSD2)," Cellular Physiology and Biochemistry, vol. 38, no. 1, pp. 83-93, 2016. 
[19] J. S. Choi, B. J. Lee, H. Y. Park et al., "Effects of donor age, longterm passage culture, and cryopreservation on tonsil-derived mesenchymal stem cells," Cellular Physiology and Biochemistry, vol. 36, no. 1, pp. 85-99, 2015.

[20] G. C. Park, J. S. Song, H. Y. Park et al., "Role of fibroblast growth factor-5 on the proliferation of human tonsil-derived mesenchymal stem cells," Stem Cells and Development, vol. 25, no. 15, pp. 1149-1160, 2016.

[21] M. Mizuta, S. Hirano, N. Hiwatashi et al., "Effect of astaxanthin on vocal fold wound healing," Laryngoscope, vol. 124, no. 1, pp. E1-E7, 2014.

[22] M. Mizuta, S. Hirano, N. Hiwatashi et al., "Effect of AST on age-associated changes of vocal folds in a rat model," Laryngoscope, vol. 124, no. 10, pp. E411-E417, 2014.

[23] R. C. Branski, B. Saltman, L. Sulica et al., "Cigarette smoke and reactive oxygen species metabolism: implications for the pathophysiology of Reinke's edema," Laryngoscope, vol. 119, no. 10, pp. 2014-2018, 2009.

[24] A. Valle-Prieto and P. A. Conget, "Human mesenchymal stem cells efficiently manage oxidative stress," Stem Cells and Development, vol. 19, no. 12, pp. 1885-1893, 2010.

[25] M. Nita and A. Grzybowski, "The role of the reactive oxygen species and oxidative stress in the pathomechanism of the age-related ocular diseases and other pathologies of the anterior and posterior eye segments in adults," Oxidative Medicine and Cellular Longevity, vol. 2016, Article ID 3164734, 23 pages, 2016.

[26] J. K. Hansen and S. L. Thibeault, "Current understanding and review of the literature: vocal fold scarring," Journal of Voice, vol. 20, no. 1, pp. 110-120, 2006.

[27] A. Branco, A. Todorovic Fabro, T. M. Goncalves, and R. H. Garcia Martins, "Alterations in extracellular matrix composition in the aging larynx," Otolaryngology and Head and Neck Surgery, vol. 152, no. 2, pp. 302-307, 2015.

[28] T. Ohno, S. Hirano, and B. Rousseau, "Age-associated changes in the expression and deposition of vocal fold collagen and hyaluronan," The Annals of Otology, Rhinology, and Laryngology, vol. 118, no. 10, pp. 735-741, 2009.

[29] Y. Cui, N. Xu, W. Xu, and G. Xu, "Mesenchymal stem cells attenuate hydrogen peroxide-induced oxidative stress and enhance neuroprotective effects in retinal ganglion cells," In Vitro Cellular \& Developmental Biology Animal, vol. 53, no. 4, pp. 328-335, 2017. 